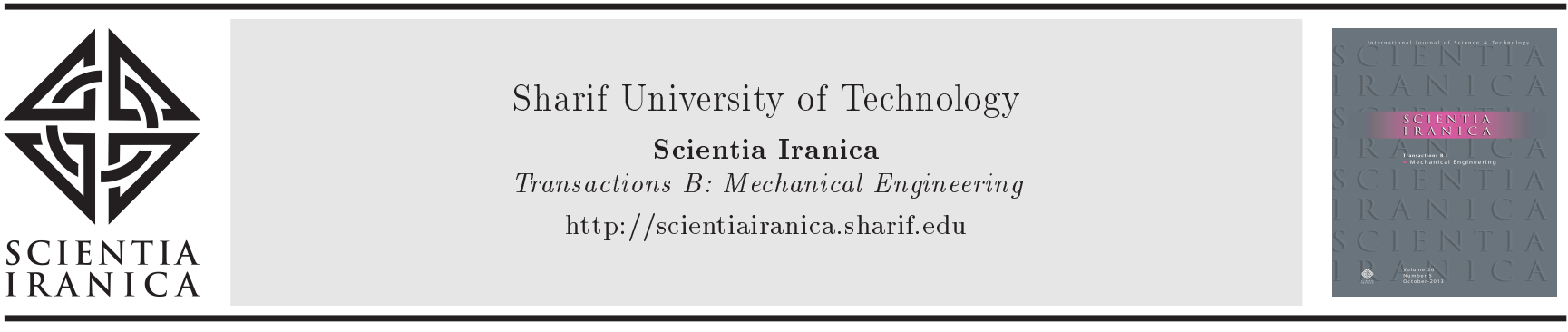

\title{
Numerical and experimental investigation of impinging turbulent flow of twin jets against a wall
}

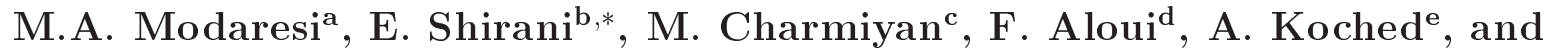 \\ M. Pavageau ${ }^{\mathrm{f}}$ \\ a. Department of Mechanical Engineering, Tarbiat Modares University, Tehran, P.O. Box 1411713116, Iran. \\ b. Department of Mechanical Engineering, Foolad Institute of Technology, Fooladshahr, Isfahan, P.O. Box 8491663763, Iran. \\ c. Department of Mechanical Engineering, University of Ayatollah Ozma Boroujerdy, Boroujerd, P. O. Box 6919969411, Iran. \\ d. LAMIH, CNRS UMR 8201, University of Valenciennes and Hainaut-Cambrésis (UVHC), Campus Mont Houy, Building \\ Gromaire, F-59313, Valenciennes Cedex 9, France. \\ e. TSI France, Hotel Technologique, Technopôle de Château-Gombert, 13382 Marseille, France. \\ f. rue de la houssiniére BP 92208, 44322 Nantes Cedex 03, France.
}

Received 30 January 2018; received in revised form 11 June 2018; accepted 15 September 2018

\section{KEYWORDS}

Impinging jets;

Twin-jet;

RANS;

Turbulence models;

PIV.

\begin{abstract}
In the present study, impinging of vertical twin jet against a horizontal plate is investigated numerically and experimentally. Four two-equation RANS based turbulence models are used and their capabilities to simulate such complicated turbulent flow are examined. The inlet jet hydraulic diameters are the same, and the Reynolds number of external flows of jets is 13500 . The ratio of the width of nozzles $(e)$ to nozzle-to-plate distance $(H)$ is considered as 1:10. The obtained results were compared with each other and two-dimensional experimental data to evaluate the capabilities of such models for this kind of flows. By comparing the numerical and experimental results, it was concluded that all of the models could predict acceptable results in the free jet area; however, in the near wall region, none of the models can predict flow characteristics with reasonable accuracy. According to the observations, at low nozzle-to-plate distances, the results of the turbulence models were approximately in accordance with the experimental data. Furthermore, maximum and minimum of the turbulent kinetic energy occurred in the shear layers and potential cores of the jets, respectively.
\end{abstract}

(C) 2019 Sharif University of Technology. All rights reserved.

\section{Introduction}

The impinging turbulent flow of twin jets has many applications due to their manifold features. They are able to mix two flows with different climatic characteristics. These kinds of jets are composed of one or several parallel air streams generally. They are commonly used in (a) the electronics cooling field, (b) stopping or slowing down heat and smoke propagation

\footnotetext{
*. Corresponding author.

E-mail address: eshirani@gmail.com (E. Shirani)
}

in case of fire in tunnels, (c) the entrance of public and commercial buildings and shops, and (d) the conservation of refrigerated foods.

Greco et al. [1] investigated the behavior of single and twin circular synthetic jets with different nozzle-to-plate distances $(H / D)$ for Reynolds number 5100 and Strouhal number 0.024 using Particle Image Velocimetry (PIV) experimentally. They compared the location of the saddle points in both configurations and found the saddle point in the single jet reaching the impinging plate; however, saddle points in the twin jet did not reach the impinging plate due to the presence of other impinging jets. In another study, Ozmen [2] examined the flow characteristics of the twin jet for 
different Reynolds numbers, nozzle-to-plate distances $(H / D)$, and jet-to-jet spacing $(L / D)$ experimentally using the smoke-wire technique. The flow structures in the impinging region using PIV method were studied by Loubiere and Pavageau [3] experimentally; then, they implemented the vortex reduction method in order to reveal the 2D coherent patterns embedded in PIV and statistical analysis of the topological and energy features of these structures. Felis et al. [4] described laboratory experiments performed to investigate the ability of air curtain arrangements to prevent heat propagation in case of fire in a corridor-like geometry. They used Laser Doppler Anemometer (LDA) and fine thermocouples for velocity and temperature measurements, respectively. Finally, results showed that the longitudinal turbulent heat flux had a dominant contribution in turbulent transfers. The impinging turbulent flow of twin jets against a wall for two different configurations using RANS-based turbulence models such as $k-\varepsilon, k-\varepsilon$ realizable and $k-\omega$ models was investigated by Fernandez et al. [5]. Results showed the $k-\varepsilon$ and $k-\varepsilon$ realizable models were perfectly in agreement with the experimental results; however, $k-\omega$ could not predict the results well; however, all of these models underestimated the jet expansion rate. Lecaros et al. [6] studied the ability of double stream twin jet air curtains for confining heat in tunnels using the Fire Dynamics Simulator (FDS) code; however, Laser Doppler Velocimetry (LDV) and fine thermocouples were used for velocity and temperature measurements, respectively. Using computational fluid dynamics techniques, Taghinia et al. [7] simulated the impinging turbulent flow of twin jets against a wall using $k-\omega-$ SST-SAS model. Finally, the results showed that SSTSAS model could predict better results for low ratios of $H / D$. Greco et al. [8] investigated the effectiveness of different nozzle-to-plate distances $(H / D)$ in heat transfer in single and twin synthetic circular jets experimentally. Results showed that, for low $(H / D)$ ratios, the heat transfer was dominant; however, for high $(H / D)$ ratios, the heat transfer performed with steady and less coherent turbulent flows. ElicerCortes et al. [9] performed 2D and 3D simulations of a double stream twin jet using the RSM model, and observed that the $2 \mathrm{D}$ and $3 \mathrm{D}$ numerical results overestimated the jet expansion rate. The influence of flow instabilities on the efficiency of the twin jet air curtains using Large Eddy Simulation (LES) was examined by Rivera et al. [10]. Results exhibited that Kelvin-Helmoltz instabilities played a more important role than Gortler instabilities. Charmiyan et al. [11] studied the impinging turbulent flow of a single jet against a wall using two different Sub-Grid Scale (SGS) models: one equation and localized dynamic Smagorinsky models. They observed that both SGS models overestimated the jet expansion rate; however, the results revealed high concurrence between the localized dynamic Smagorinsky model results and the experimental data in simulating the subgrid structures in the impinging region, especially near the impingement wall. Recently, Liang $\mathrm{Xu}$ et al. [12] simulated heat transfer by swirling impinging jets issued from a screwthread nozzle due to achieved uniform cooling at high heat transfer rates. In addition, they observed that the designed swirler configuration increased the radial uniformity of heat transfer. Large eddy simulation was used by Martin Draksler et al. [13] to achieve flow dynamics and heat transfer characteristics of multiple impinging jets in hexagonal configuration. Trinh et al. [14] compared the aerodynamics and thermal heat transfer of impinging jets between lobed and tubed injections and, then, observed that heat transfer rate was very different between them. Finally, Tsaoulidis and Angeli [15] studied dispersions of two immiscible liquids in confined intensified impinging jets' cells experimentally, and found that the total velocity of the two jets was the main parameter that affected the drop sizes. Recently, Charmiyan et al. [16] investigated different structures of slot impinging jet using different criteria using tomographic PIV. They extracted some new three-dimensional futures of turbulent flow close to and far from the impinging wall. The results of Proper Orthogonal Decomposition (POD) showed that the dominant flow structures belonged to the first forty POD modes.

The present study investigates the ability of four two-equation RANS-based turbulence models such as $k-\varepsilon, k-\varepsilon-\mathrm{RNG}, k-\omega$, and $k$ - $\omega$-SST to predict the behavior of the twin jet against a wall in three different regions: near the inlets, in the middle, and near the impinging wall. Therefore, the numerical procedure and experimental setup along with its method adopted in this study are first described briefly. Finally, the numerical and experimental results are compared together from new viewpoints. The first location of the impingement of two-jet boundary layers and, also, the first location of a single jet formation obtained using numerical simulation are then compared with experimental data.

The configuration used here is shown in Figure 1. It is a symmetric non-recirculating twin jet system, whose discharge jets were supplied with the air taken from the ambience. The heat transfer phenomenon, coherent eddy structures, and different arrangements of twin jets were investigated in previous studies [3$5,9,10]$. This study differs from previous studies in terms of configuration, boundary conditions, turbulence models, and the Reynolds number of external flows of jets. In the present study, the impinging turbulent flow of twin jets against a wall using different two-equation turbulence models, $k-\varepsilon, k-\varepsilon$-RNG, $k-\omega$, and $k-\omega$-SST was simulated. Finally, the numerical and 


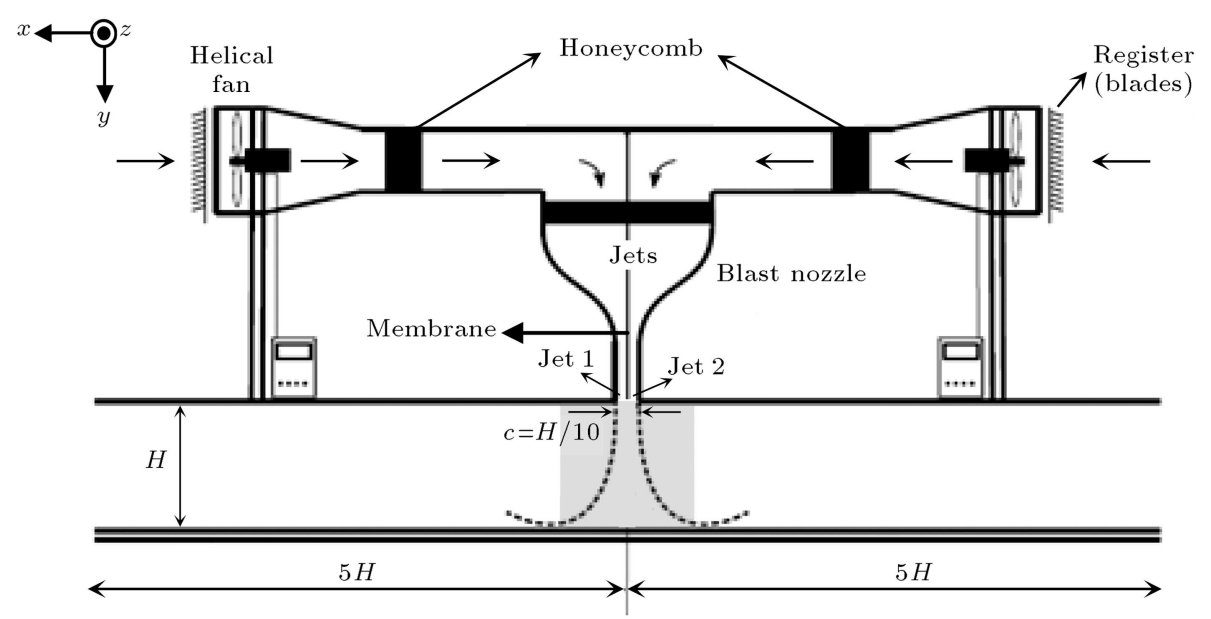

Figure 1. Schematic of the device for generating a double air jet plane impact.

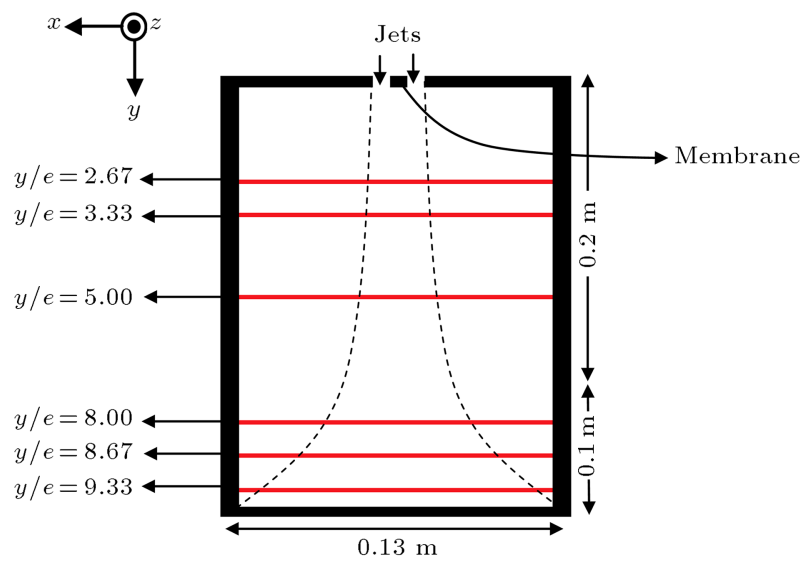

Figure 2. Different sections for comparison of numerical results against the experiments. The jet hydraulic diameter and the membrane are $3 \mathrm{~cm}$ and $2 \mathrm{~mm}$, respectively.

experimental results were compared. Figure 2 shows different sections from which experimental and numerical results were extracted. Note that dimensions of the numerical and experimental geometries were considered the same and, then, the simulation was performed for the entire domain, and only the shaded area in Figure 1 was investigated as the laboratory experiment.

\section{Numerical procedure}

2D Navier Stokes equations along with $k-\varepsilon$ standard, $k$ -
$\varepsilon$-RNG, $k-\omega$ standard, and $k$ - $\omega$-SST models were used for the $2 \mathrm{D}$ steady-state simulation. A detailed description of the governing equations related to these models was presented previously in [17]. All of the simulations were carried out using the CFD code OpenFOAM 2.3.0 package. The residuals of velocity, pressure, turbulent kinetic energy, and dissipation rate were set to $10^{-5}$ in order to obtain the convergence stability [5], and SimpleFoam solver was used for simulation. Due to the high Reynolds number, the effects of gravity were ignored; in addition, gradients, divergences, and Laplacian terms were evaluated using Gauss linear, bounded Gauss limited linear, and Gauss linear corrected methods, respectively. A detailed description of the discretizing methods for different terms can be found in $[18,19]$. In order to examine the grid size, three cases were investigated, as given in Table 1 . In all of these cases, the shaded area shown in Figure 1 consists of high density mesh, and then by moving towards outlets and the walls, the density of the mesh goes lower and higher, respectively. On the impinging wall, the density of the mesh is higher than that on the other wall.

Mean velocity and turbulence kinetic energy for these cases in different sections were compared (Figure 3), and Case 3 was chosen in the end.

\section{Boundary conditions}

The boundary conditions were considered in accordance with the experiments. At the whole wall of the

Table 1. Grid-independency tests.

\begin{tabular}{cccc}
\hline Name of cases & Number of cells & $\begin{array}{c}\text { Turbulence kinetic energy } \\
\left(\mathbf{m}^{\mathbf{2}} / \mathbf{s}^{\mathbf{2}}\right)\end{array}$ & $\begin{array}{c}\text { Velocity } \mathbf{y} / \mathbf{e}=\mathbf{8 . 6 7}) \text { at } \\
\boldsymbol{y} / \boldsymbol{e}=\mathbf{8 . 6 7}\end{array}$ \\
\hline Case 1 & 76572 & 0.35 & 4.98 \\
Case 2 & 151812 & 0.052 & 5.1 \\
Case 3 & 300852 & 0.05 & 5.1 \\
\hline
\end{tabular}




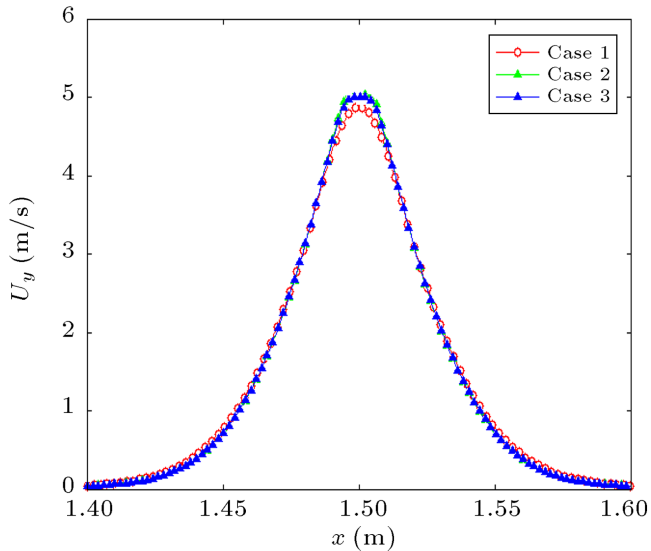

(a)

Figure 3. Grid-independency tests at $y / e=8.67$ :

geometry, a no-slip condition was imposed. Further, at the inlets and outlets of the geometry, a mean velocity equal to $7 \mathrm{~m} / \mathrm{s}$ and a constant pressure equal to the atmospheric pressure were imposed, respectively. The application of PIV data determined the turbulent kinetic energy at the inlets. Moreover, at the inlets, the value of dissipation rate $(\varepsilon)$ and specific dissipation rate $(\omega)$ are taken equal to $\varepsilon=C_{\mu}^{3 / 4} K^{3 / 2} / 0.07 b(b=e / 2$, $\left.C_{\mu}=0.085\right)$ and $\omega=K^{1 / 2} / 0.07 C_{\mu}^{1 / 4}$ for $k-\varepsilon, k-\varepsilon-\mathrm{RNG}$, $k-\omega$, and $k-\omega$-SST models, respectively.

\section{Description of the experimental setup}

A schematic representation of the experimental setup is presented in Figure 1, and a detailed view of the test section is shown in Figure 4 . The test bench consists of an open tunnel with $1 \times 0.3 \mathrm{~m}^{2}$ on both sides and a total length equal to $3 \mathrm{~m}$. In the middle and at the top of the tunnel, two continuous rectangular nozzles are placed. To ensure uniform flow at the nozzle exits, the honeycomb structures are used for homogenization; then, the jets emerge by two rectangular nozzles with a length and a width equal to $1 \mathrm{~m}$ and $1.5 \mathrm{~cm}$ (Figure 1),

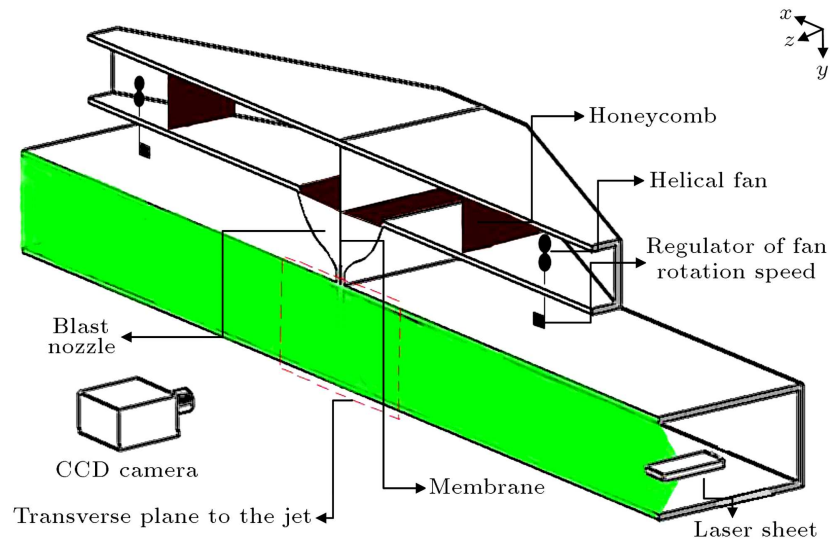

Figure 4. Plan measures by PIV.

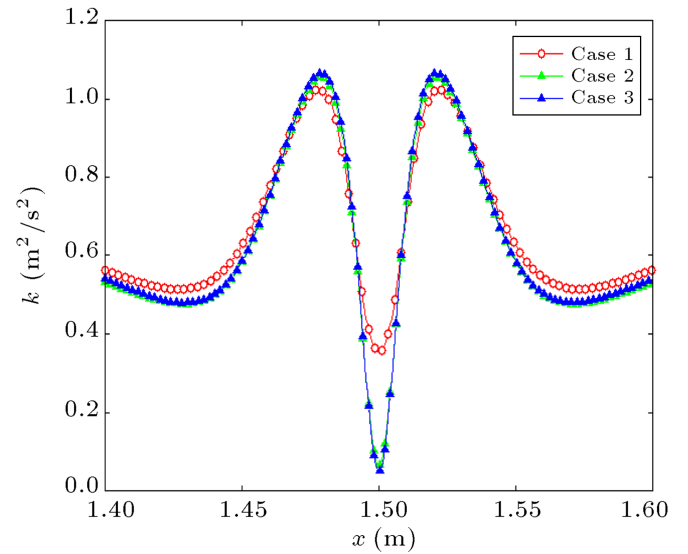

(b)

(a) Streamwise profiles and (b) turbulent kinetic energy.

respectively. A metal plate with a thickness of $2 \mathrm{~mm}$ is used to separate two jets. The blowing velocity (i.e., the velocity of the jet at the nozzle exit) can be set separately for each nozzle, and their range changes from $1 \mathrm{~m} / \mathrm{s}$ to $15 \mathrm{~m} / \mathrm{s}$ by varying the rotational speeds of the axial fans. The blower velocity is set to $7 \mathrm{~m} / \mathrm{s}$. The associated Reynolds number, $\operatorname{Re}=\frac{U_{0} e}{\nu}$, is 13500 , where $U_{0}$ is the mean velocity at the nozzle exit, $e$ is the total width of nozzles, their half width is $b$ $(e=2 b=3 \mathrm{~cm})$, and $\nu$ is the kinematic viscosity of air. Test section dimensions are adjusted with respect to the following aspects of relationships: $H / e=10$ for maximum turbulent intensity on the jet axis and $L_{1} / e>=20\left(L_{1}\right.$ is the tunnel depth) to ensure the two-dimensionality of the flow.

\section{The PIV measurements}

The experimental data are obtained by fast PIV measurements in a transverse plane to the jet (Figure 4). A high-frequency acquisition system is used because of the high frequency of the phenomena that occurred. Measurements by fast PIV are performed using a system marketed by LaVision. For a good resolution of the total velocity field of the flow, PIV measurements were considered in 9 areas of the jet, as shown in Figure 5. The size of each measurement plane $x-y$ is $13 \times 13 \mathrm{~cm}^{2}$. The flow is seeded with sprayed olive oil tracer particles with compressed air at 4 bar. The size of tracer particles is equal to $5 \mu \mathrm{m}$ approximately. These particles are then illuminated by $5 \mathrm{~W}$ power continuous laser, which delivers a beam of wavelength equal to $532 \mathrm{~nm}$. This beam is then diverged by means of a semi-spherical lens for generating a laser sheet of $1 \mathrm{~mm}$ thick. This laser is synchronized with a camera of high frequency, High Speed Star 6 (HSS6), which allows recording images of the flow doublets (tracer particles) at a frequency of up to $50 \mathrm{kHz}$. The resolution of the camera CCD sensor is equal to $1024 \times 1024$ pixels. 


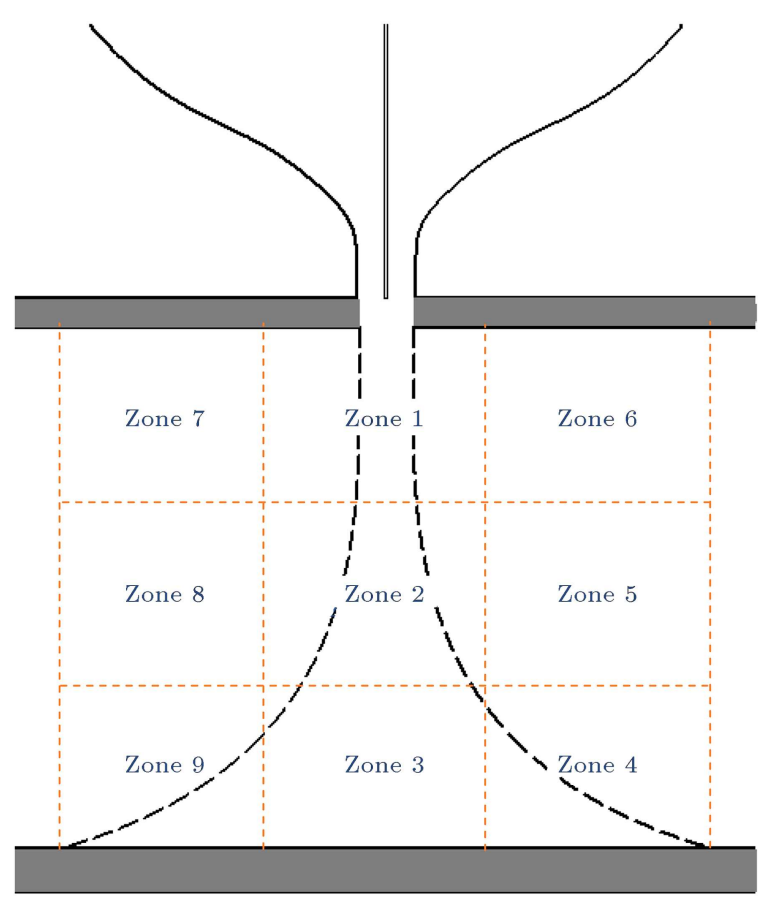

Figure 5. Areas of PIV measurements.

The camera is equipped with a Nikon lens 1:2.8 and a focal length of $50 \mathrm{~mm}$ placed at a distance of $30 \mathrm{~cm}$ to the measurement plane. The recorded images are then decomposed into several interrogation zones of $16 \times 16$ pixels in size, whereas there is a $50 \%$ overlap between two images of the same pair. The size of the interrogation zone is adjusted adaptively to allow for a better estimate of the velocity of the flow. A processing algorithm based on the method of cross-correlation between the images of the same pair is used to estimate the probability density function of the particle displacement in the same interrogation zone and, subsequently, to estimate the velocity vector in each interrogation zone considering the size of the last metric in length. To increase the number of velocity vectors, an analytical method for considering a 50\% overlap between two areas of the same pair is used. Then, 12500 snapshots are used in order to obtain the mean velocity fields and fluctuating quantities. Table 2 summarizes the fixed parameters for the use of fast PIV.

\section{Results and discussion}

Figure 6(a)-(d) show the numerical results of mean velocity contours that have been obtained using four different turbulence models. All of them, except $k-\omega$ model, can predict the location of the formation of a single jet, where the two jets merge into one another, acting as a single jet (highlighted with an ellipse in Figure 6(c). The weakness of the $k-\omega$ model is related to its inability in a free jet problem, because it could not predict the diffusion phenomenon well. The narrow area between the two jets is where they gradually merge together. After the complete integration of the two jets (formation of a single jet), the narrow area disappears. However, before the complete integration of the two jets, the fluid between the two jets is pulled into the jets due to shear stress; therefore, velocity in this region is lower than that in the jet center. Moreover, Turbulent Kinetic Energy (TKE) contours are presented in Figure 7. All models predicted a similar procedure of variations for TKE. The main difference between the aforementioned models lies in predicting TKE in the impingement region. The $k-\varepsilon$ and $k-\varepsilon-\mathrm{RNG}$ models predict about $1.6 \mathrm{~m}^{2} / \mathrm{s}^{2}$ and $1.1 \mathrm{~m}^{2} / \mathrm{s}^{2}$ for TKE in this region, respectively (the parts are shown in ellipses in Figure 7(a) and (b), while two other models predict much lower values in the impingement region (about $0.1 \mathrm{~m}^{2} / \mathrm{s}^{2}$ ). Predictions similar to our predictions for TKE were reported in [17].

Table 2. Characteristics of PIV acquisitions.

\begin{tabular}{lc}
\hline Focal length of the lens (mm) & 50 \\
Dimension measurement plan $\left(\mathrm{mm}^{2}\right)$ & $130 \times 130(\approx 4 e \times 4 e)$ \\
CCD sensor resolution (pixels) & $1024 \times 1024$ \\
Size of the interrogation zone (pixels) & $32 \times 32$ \\
Size of the interrogation zone $\left(\mathrm{mm}^{2}\right)$ & $4 \times 4(\approx e / 8 \times e / 8)$ \\
Overlap between the interrogation zones & $50 \%$ \\
Number vector in the velocity field & $64 \times 64$ \\
Spatial resolution of the velocity vectors $(\mathrm{mm})$ & $2(\approx e / 15)$ \\
Measurement accuracy for a confidence interval of $95 \%$ & $\pm 5 \%$ \\
Acquisition frequency (Hz) & 2500 \\
Number of pairs of recorded images & 5000 \\
\hline
\end{tabular}




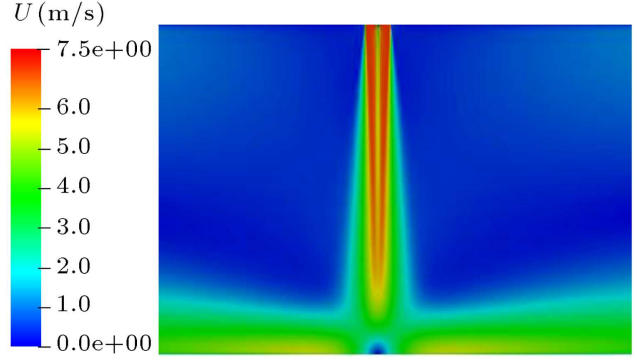

(a)

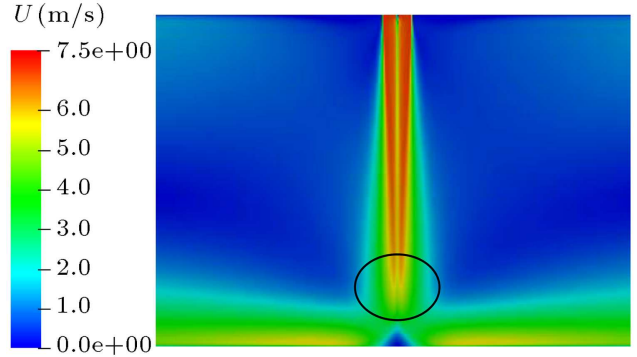

(c)

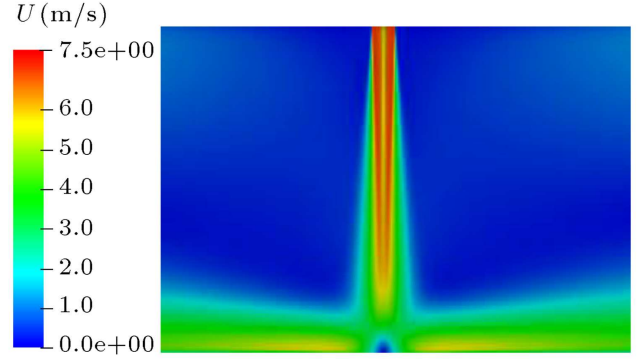

(b)

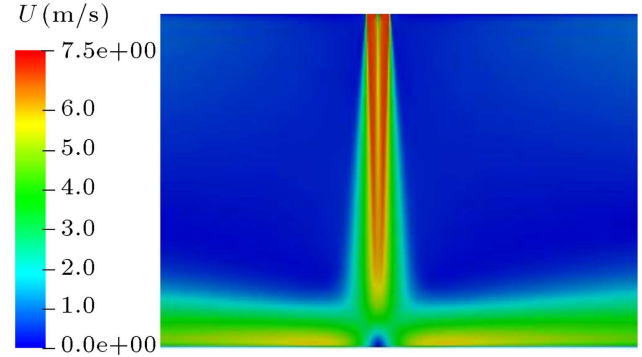

(d)

Figure 6. Contours of mean velocity magnitude using different turbulence models: (a) $k-\varepsilon$, (b) $k-\varepsilon-\mathrm{RNG}$, (c) $k-\omega$, and (d) $k-\omega-\mathrm{SST}$.
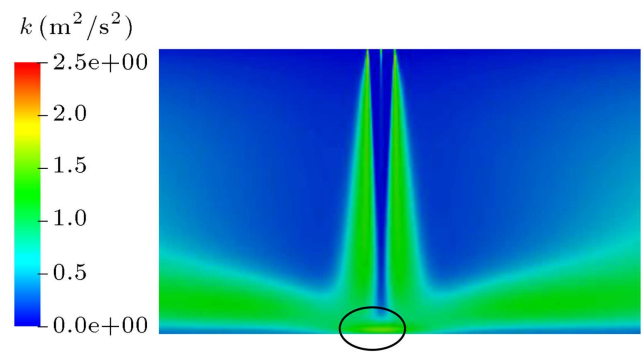

(a)
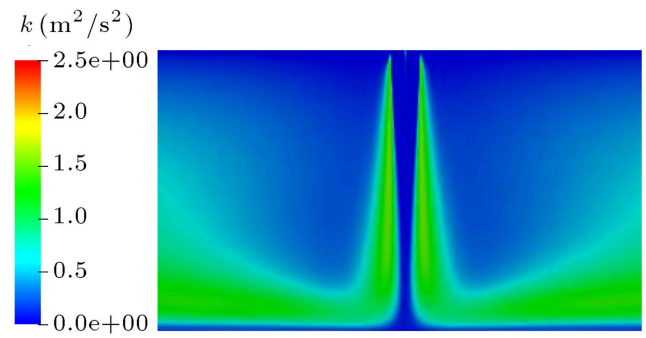

(c)

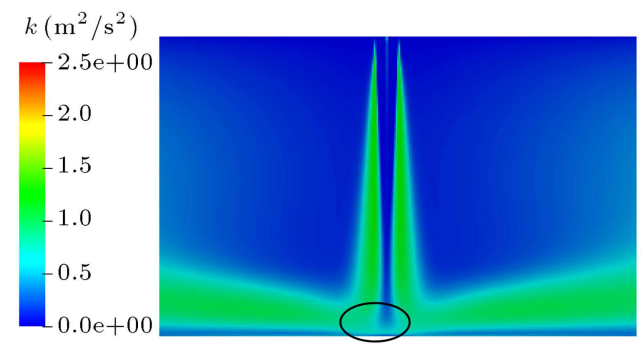

(b)

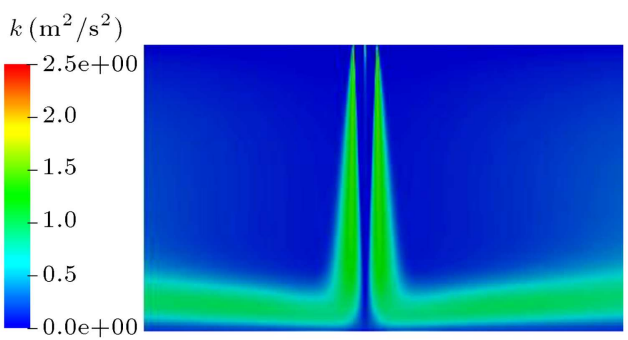

(d)

Figure 7. Contours of turbulence kinetic energy using different turbulence models: (a) $k-\varepsilon$, (b) $k-\varepsilon-\mathrm{RNG}$, (c) $k-\omega$, and (d) $k-\omega-\mathrm{SST}$.

Figure 8(a)-(f) present experimental data and numerical simulation results of the four aforementioned turbulence models for streamwise profile variations in different horizontal sections. Once the fluid jet leaves the two nozzles, shear layers of the two jets appear and generate some instabilities. As the flow gets closer to the impinging wall, the two jets would have further entrained with each other; therefore, at $y / e=7$, the two jets behave as if they are a single jet with the maximum velocity at the center of the jet. From this point on, the flow behavior can be accounted for as that of a single jet. As the flow goes further close to the impinging wall, the stagnation flow area appears and vertical velocity at the center of the jet is reduced, making a smoother vertical velocity profile.

By comparing the four different turbulence models, it was observed that, at $y / e=2.67$ and $y / e=3.33$ sections, the prediction results of the turbulence models were approximately in accordance with the experimental data, particularly for a zone near the midline separating the two jets (Figure $8(\mathrm{a})$ and (b)). For zones away from the midline $(|x / e|>1)$, where no dominant 


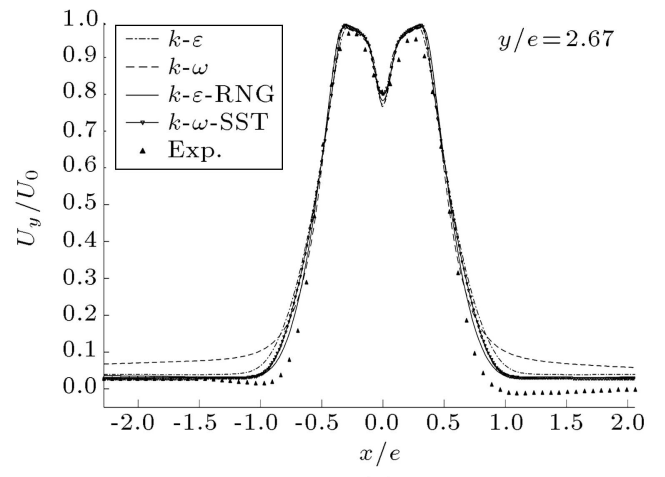

(a)

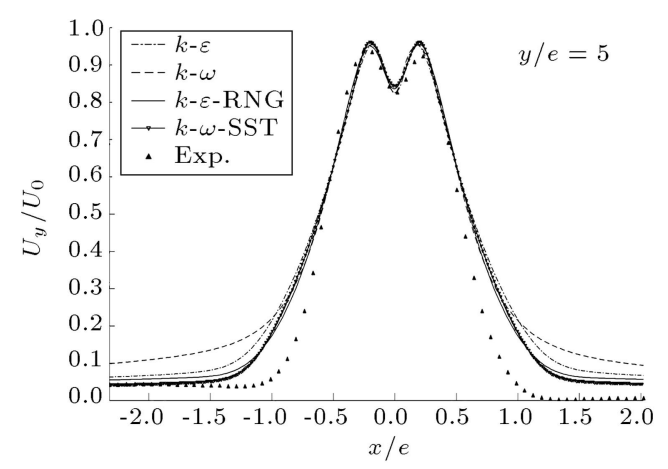

(c)

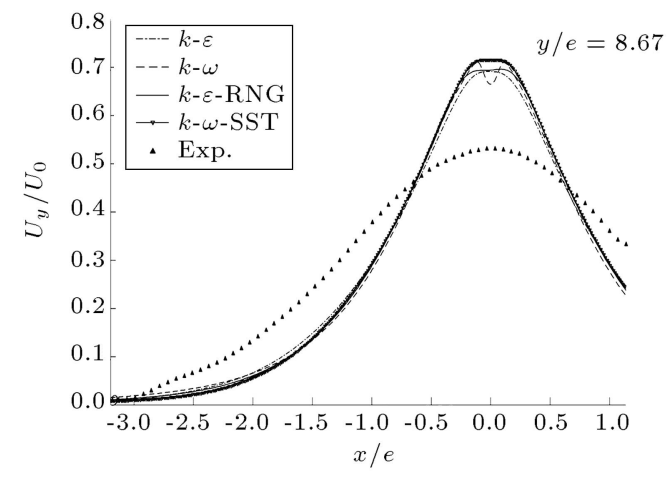

(e)

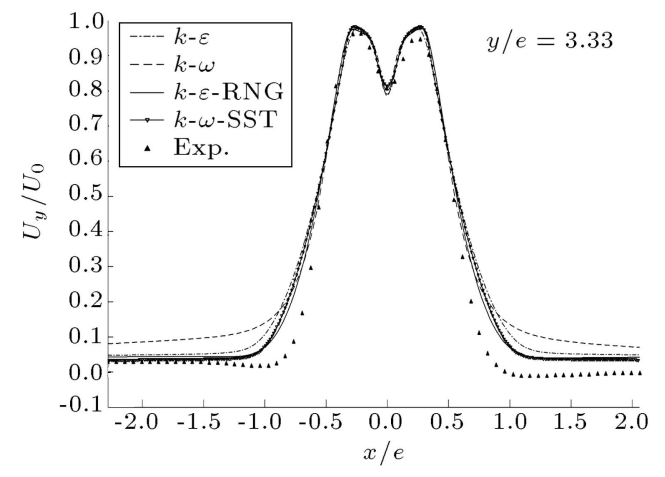

(b)

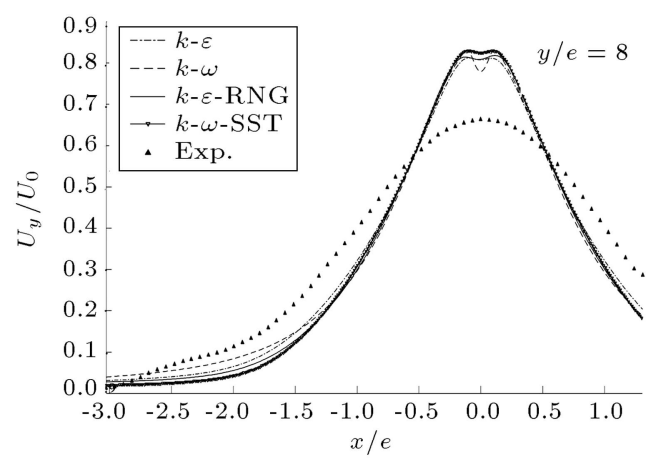

(d)

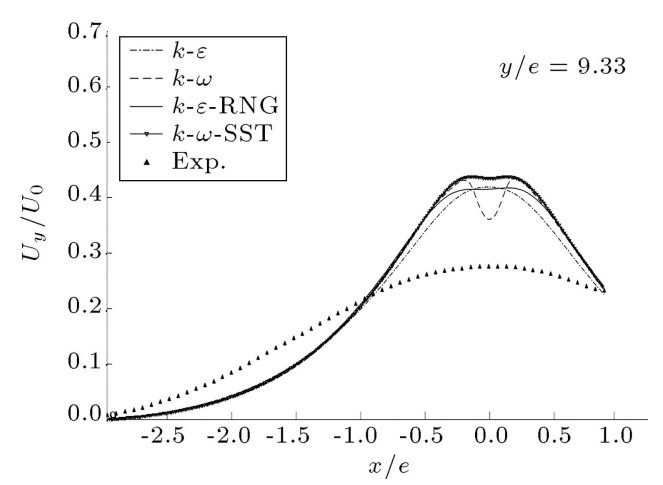

(f)

Figure 8. Streamwise profiles at different sections along $x$ direction for different turbulence models and experimental data.

flow is identified and velocities are much smaller than those of the zone near the midline $(|x / e|<1)$, the results obtained by $k-\varepsilon-\mathrm{RNG}$ and $k-\omega$-SST models, compared to those of the standard $k-\varepsilon$ and $k-\omega$ models, are highly in agreement with the experimental data. For the $k-\varepsilon-\mathrm{RNG}$ model, the modifications applied to the standard $k-\varepsilon$ model lead to a modified model, considering different length scales when calculating the diffusion term so that one could end up with more accurate diffusion term calculations of the shear layer area and beyond; this results in highly accurate velocity calculations at farther distances from the midline, where smaller turbulent structures are dominant.

Figure 9 shows the TKE at different sections. As shown in Figure 9(a)-(c), for the distance starting from the point at which the fluid leaves the nozzle and extending up to $y / e=5$, TKE is at its maximum and minimum values in shear layers $(0.4<|x / e|<$ 0.7 ) and potential cores of the jets, respectively. For the shear layers, the best and worst TKE predictions are found to be those of RNG and $k-\omega$ methods, respectively. Going farther from the midline of the jets, the results of $k-\omega$-SST method tend to gradually show the highest accordance with experimental data. In the area between the two jets where the shear layers of the two jets impinge against one another, due to the formation of Kelvin-Helmholtz instabilities, TKE increases slightly. Within this area, except for the $k-\omega$ model, the results of all models are almost identical and TKE is underestimated with reference to the experimental 


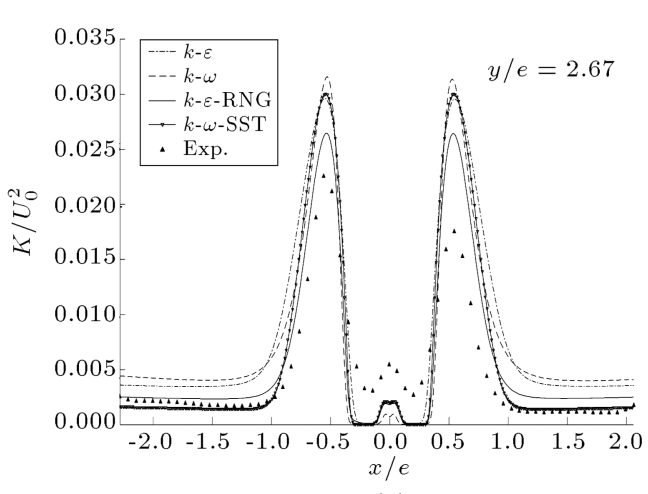

(a)

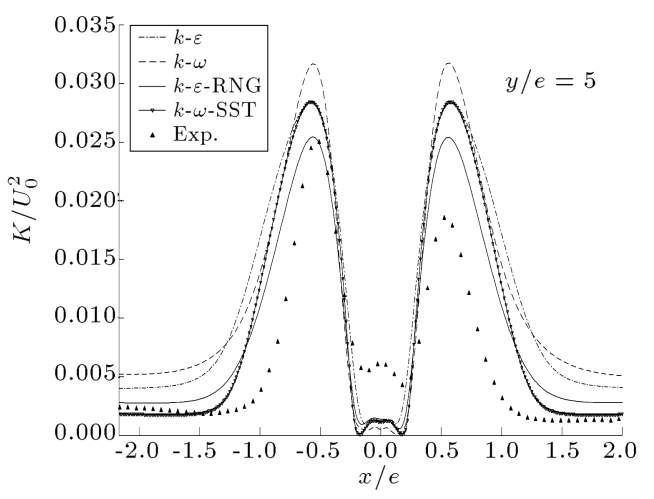

(c)

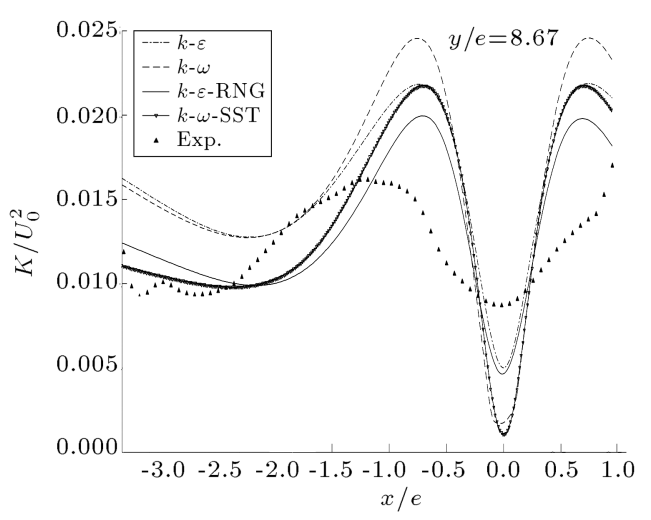

(e)

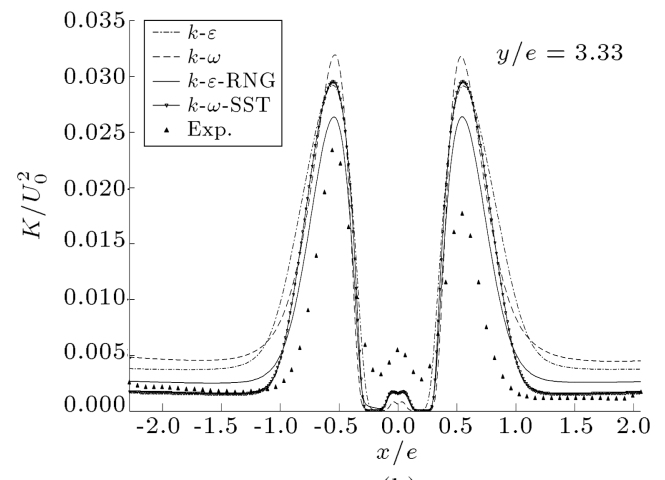

(b)

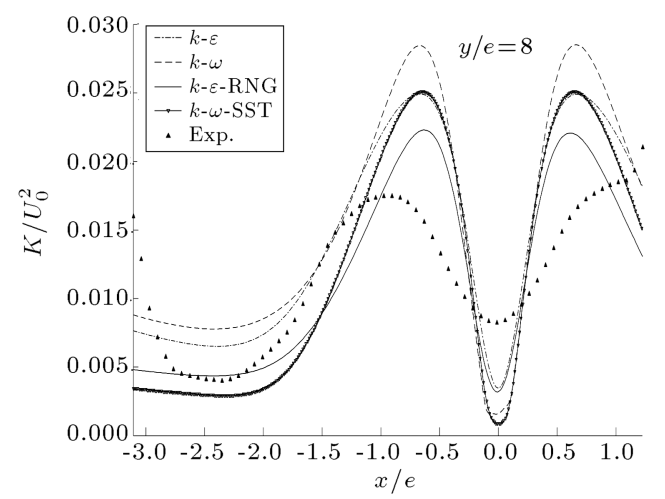

(d)

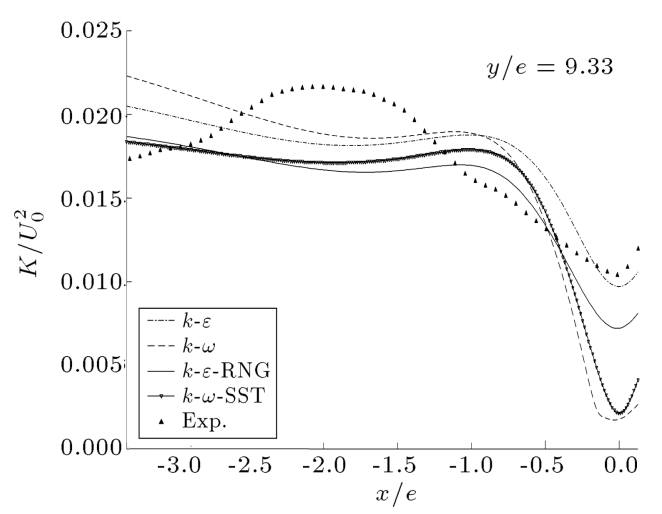

(f)

Figure 9. Turbulence kinetic energy profiles at different sections along $x$ direction for different turbulence models and experimental data.

data. The difference between experimental data and simulation results increased at farther distances from the jet nozzles. For the investigated models, the highest error values associated with $k-\varepsilon, k-\varepsilon-\mathrm{RNG}, k-\omega$, and $k$ $\omega$-SST models within the free jet area and for $y / e=5$, $|x / e|<0.2$ are calculated to be $73.44,73.12,86.67$, and $73.44 \%$, respectively. These values have been obtained using Eq. (1):

$$
\begin{aligned}
\text { error }= & \frac{\left|(y / e)_{\max , \text { experimental }}-(y / e)_{\max , \text { numerical }}\right|}{(y / e)_{\max , \text { experimental }}} \\
& \times 100 .
\end{aligned}
$$

At farther distances (i.e., $y / e>8$, Figures (8)d-(f) and $9(\mathrm{~d})-(\mathrm{f}))$, the two jets merge into one another, acting as a single jet. In this area, due to the existence of the potential core of the jet, the jet flow at $|x / e|<0.5$ shows minimum turbulence intensity, and TKE value is maximum at the two boundary layers, which are formed on the two sides of the jet. As be seen in Figures $8(\mathrm{~d})$-(f) and $9(\mathrm{~d})-(\mathrm{f}), k-\varepsilon-\mathrm{RNG}$ model is closest to the experimental data, both within the jet's core area and at the single jet's shear layer. At near wall areas where $|x / e|<0.2$, the flow incurs the largest normal strain as it touches the wall and returns vertically. The obtained results indicate that the $k-\varepsilon$ and $k$ - $\varepsilon$-RNG models behave better, while the $k-\omega$-SST model has the largest errors in estimating TKE in this 
area $(|x / e|<0.2)$. Compared to the $k-\varepsilon$ model, the $k-\varepsilon-$ RNG model is found to enjoy higher accuracy for shear layer areas. The largest error values are associated with the $k-\varepsilon, k-\varepsilon$-RNG, $k$ - $\omega$, and $k$ - $\omega$-SST models within the single jet area; in addition, for $y / e=9.33,|x / e|<0.2$, they are calculated to be $8.6,30.5,81$, and $78.1 \%$, respectively. These values are obtained using Eq. (1).

At farther distances from the midline, near the wall, what is referred to as wall jet develops. Within this area, the jet's vertical velocity transforms into near-wall horizontal velocity as it touches the wall, developing a boundary layer. As a result, turbulence intensity increases within this area (Figure 8(f)). In the wall-jet area, the best TKE predictions are once more obtained through the $k$ - $\varepsilon$-RNG model. Closely following the $k$ - $\omega$-SST model, the $k-\varepsilon$ model is also better than $k-\omega$.

Figure 10 shows the general structures of the streamlines of mean flow in impinging twin jets. In order to further compare the models, the position where the primary vortex was developed along the flow was both experimentally and numerically studied. Figure 11 shows the obtained streamlines using $k$ - $\varepsilon$ RNG model for half of the solution domain. As can be seen, as the flow touches the lower wall and returns upward and considering the confinement of the twin jet flow impinging against the wall, a primary, large vortex develops, around which the average flow circulates. The comparison between the predicted vortex center and the experimental data indicates that the results of $k$ - $\omega$-SST, $k-\varepsilon$-RNG, $k-\varepsilon$, and $k$ - $\omega$ (expressed in order of decreasing accordance) are in good agreement with the experimental data, respectively (see Table 3 ).

When the two jets leave the nozzles, first, their boundary layers impinge against one another (Figure 12). Table 4 presents a comparison between the first impinging position of the boundary layers of

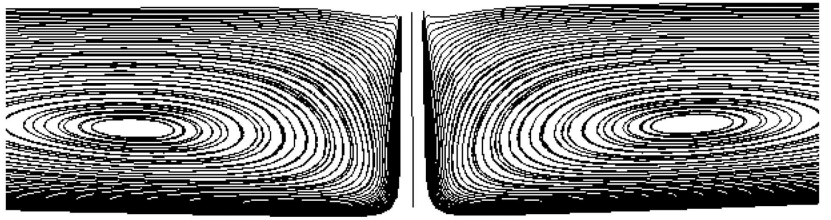

Figure 10. Streamlines of mean flow using $k-\omega$ to investigate the center of the main vortex.

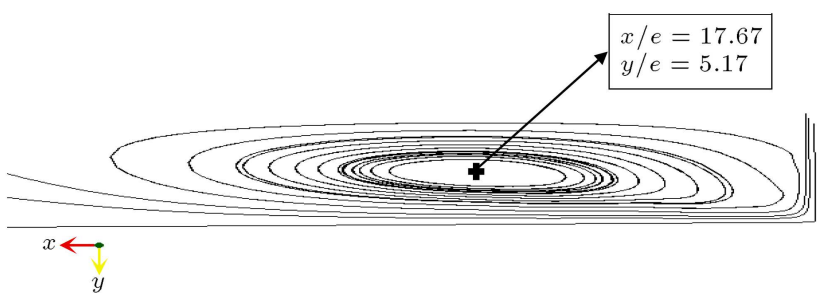

Figure 11. Coordinates of the center of the main vortex obtained from $k-\varepsilon-\mathrm{RNG}$ model.
Table 3. Coordinates of the center of the main vortex obtained by different turbulence models.

\begin{tabular}{lcc}
\hline \multirow{2}{*}{ Models } & \multicolumn{2}{c}{ Coordinates } \\
\cline { 2 - 3 }$k-\varepsilon$ & $\boldsymbol{x} / \boldsymbol{e}$ & $\boldsymbol{y} / \boldsymbol{e}$ \\
$k-\varepsilon-\mathrm{RNG}$ & 14.67 & 5.33 \\
$k-\omega$ & 17.67 & 5.17 \\
$k-\omega-\mathrm{SST}$ & 12.33 & 5.33 \\
Experiment & 22 & 5.5 \\
& 18.23 & 5.43 \\
\hline
\end{tabular}

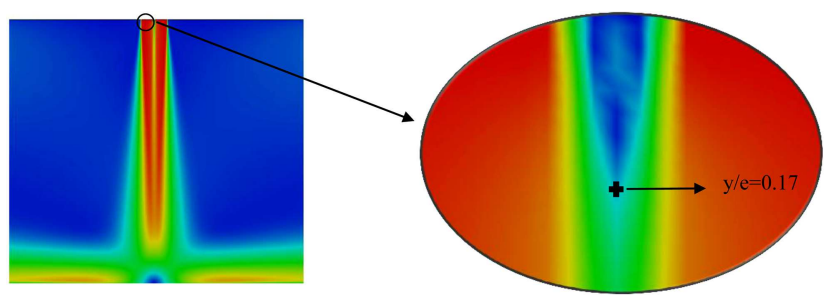

Figure 12. Location of coinciding boundary layers of two jets obtained by $k-\varepsilon-\mathrm{RNG}$ model.

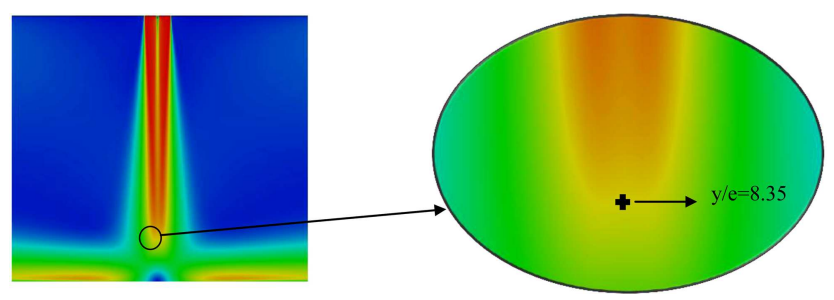

Figure 13. Location of merging two initial jets using $k$ - $\varepsilon$-RNG model.

the jets as predicted by different numerical models and those measured on experimental data. As can be observed, the results of $k$ - $\varepsilon$-RNG model represent better estimations in terms of the extension of shear layers of the jets and their integration. Eq. (1) is used to compute the differences given in Table 4 .

Another comparison made concerning the results of numerical models is focused on the point at which the two jets fully merge to form a single jet (Figure 13). Table 5 shows the position at which the two jets merge, as estimated by different numerical models and numerical results. As can be inferred from the results, three models $(k-\omega$-SST, $k-\varepsilon$-RNG, and $k-\varepsilon)$ showed relatively similar results, even though $k-\varepsilon$ model was associated with slightly more accurate results than the other two models. The important point is that the $k$ $\omega$ model is unable to model the merging of the two jets, predicting separate jets up to a position close to the wall. Based on the results reported in Table 5, the proposed numerical models in the present paper overestimated the distance from the jet nozzle existing at which the two jets merged to form a single jet; this is because of the incapability of the models when it 
Table 4. Location of the concurrent boundary layers of two jets obtained by different turbulence models.

\begin{tabular}{lcc}
\hline Models & $\begin{array}{c}\text { Location of coinciding } \\
\text { boundary layers of two jets } \\
(\boldsymbol{y} / \boldsymbol{e})\end{array}$ & Differences (\%) \\
\hline$k-\varepsilon$ & 0.15 & 10.2 \\
$k-\varepsilon-\mathrm{RNG}$ & 0.17 & 1.8 \\
$k-\omega$ & 0.18 & 7.8 \\
$k-\omega-\mathrm{SST}$ & 0.16 & 4.2 \\
Experiment & 0.167 & - \\
\hline
\end{tabular}

Table 5. Location of merging two initial jets using different turbulence models.

\begin{tabular}{lcc}
\hline Models & $\begin{array}{c}\text { Location of } \\
\text { merging two } \\
\text { initial jets }(\boldsymbol{y} / \boldsymbol{e})\end{array}$ & Differences (\%) \\
\hline$k-\varepsilon$ & 8.33 & 19 \\
$k-\varepsilon-\mathrm{RNG}$ & 8.35 & 19.3 \\
$k-\omega$ & - & - \\
$k-\omega-\mathrm{SST}$ & 8.5 & 21.4 \\
Experiment & 7 & - \\
\hline
\end{tabular}

comes to decelerating region simulation due to large pressure gradients and overestimated velocities within the region. Eq. (1) is used to compute the differences given in Table 5 .

As can be seen from the results shown in Figures 10 and 11, there are rather large differences between the simulated results and experimental results. The reason is related to turbulence modeling. It seems that the two-equation models are not capable to correctly and accurately predict such flows due to high curvature of streamlines, the existence of shear layers and large pressure gradients, and also backflow in the impinging turbulent flow of twin jets.

\section{Conclusion}

In the present paper, impinging turbulent twin jet air flowing at a Reynolds number of 13500 and at a nozzle-to-plate distance of 10 times the total width of nozzles (20 times the width of each nozzle) was twodimensionally simulated using four turbulence models: standard $k-\varepsilon, k-\varepsilon-\mathrm{RNG}, k-\omega$, and $k-\omega$-SST models. The four models were used to predict average velocity, kinetic energy, primary flow vortex center, the point at which shear layers of the two jets impinge against one another, and the position where the two jets merge to form a single jet. The results were then compared against two-dimensional PIV experimental data, which were also undertaken by the authors.

The results indicated that all four models were capable to simulate the flow within a free jet region.
However, in an area closer to the impinging wall, the differences between the experimental data and simulated results increased gradually. At the moment when the distance at which the two jets merged into a single jet, the best predictions were those obtained by $k$ - $\varepsilon$-RNG model, and the worst results corresponded to the $k-\omega$ model.

\section{Nomenclature}

e Total width of the twin jet $(\mathrm{m})$

$H \quad$ Tunnel height $(\mathrm{m})$

$L \quad$ Tunnel length $[10 H](\mathrm{m})$

Re The jet Reynolds number (for each stream)

$U_{x} \quad$ Mean velocity in the $x$ direction $\left(\mathrm{ms}^{-1}\right)$

$U_{y} \quad$ Mean velocity in the $y$ direction $\left(\mathrm{ms}^{-1}\right)$

$U_{0} \quad$ Velocity at the nozzle exit $\left(\mathrm{ms}^{-1}\right)$

$K \quad$ Turbulence kinetic energy $\left(\mathrm{m}^{2} \mathrm{~s}^{2}\right)$

$D \quad$ Diameter of each nozzle [1]

$x, y, z \quad$ Cartesian coordinate system (m)

\section{Abbreviations}

TKE Turbulence Kinetic Energy

RANS Reynolds-Averaged Navier-Stokes

PIV Particle Image Velocimetry

LDA Laser Doppler Anemometer

FDS Fire Dynamics Simulator

LDV Laser Doppler Velocimetry

\section{References}

1. Greco, C., Castrillo, G., Crispo, C., Astarita, T., and Cardone, G. "Investigation of impinging single and twin circular synthetic jets flow field", Experimental Thermal and Fluid Science, 74, pp. 354-367 (2016).

2. Ozmen, Y. "Confined impinging twin air jets at high Reynolds numbers", Experimental Thermal and Fluid Science, 35, pp. 355-363 (2011). 
3. Loubiere, K. and Pavageau, M. "Educing coherent eddy structures in air curtain systems", Chemical Engineering and Processing: Process Intensification, 47, pp. 435-448 (2008).

4. Felis, F., Pavageau, M., Elicer-Cortes, J.C., and Dassonville, T. "Simultaneous measurements of temperature and velocity fluctuations in a double streamtwin jet air curtain for heat confinement in case of tunnel fire", International Communications in Heat and Mass Transfer, 37, pp. 1191-1196 (2010).

5. Fernandez, J., Elicer-Cortes, J.C., Valencia, A., Pavageau, M., and Gupta, S. "Comparison of low-cost two-equation turbulence models for prediction flow dynamics in twin-jets devices", International Communications in Heat and Mass Transfer, 34, pp. 570-578 (2007).

6. Lecaros, M., Elicer-Cortes, J.C., Fuentes, A., and Felis, F. "On the ability of twin jets air curtains to confine heat and mass inside tunnels", International Communications in Heat and Mass Transfer, 37, pp. 970-977 (2010).

7. Taghinia, J., Rahman, M.M., and Siikonen, T. "Numerical investigation of twin-jet impingement with hybrid-type turbulence modeling", Applied Thermal Engineering, 73, pp. 650-659 (2014).

8. Greco, C.S., Ianiro, A., and Cardone, G. "Time and phase average heat transfer in single and twin circular synthetic impinging air jets", International Journal of Heat and Mass Transfer, 73, pp. 776-788 (2014).

9. Elicer-Cortes, J.C., Demarco, R., Valencia, A., and Pavageau, M. "Heat confinement in tunnels between two double-stream twin-jet air curtains", International Communications in Heat and Mass Transfer, 36, pp. 438-444 (2009).

10. Rivera, J., Elicer-Cortes, J.C., and Pavageau, M. "Turbulent heat and mass transfer through air curtains devices for the confinement of heat inside tunnels", International Communications in Heat and Mass Transfer, 38, pp. 688-695 (2011).

11. Charmiyan, M., Azimian, A., Keirsbulc, K.L., Shirani, E., and Aloui, F. "Turbulent plane impinging jetphysical insight and turbulence modeling", Journal of Applied Fluid Mechanics, 9, pp. 11-17 (2016).

12. Xu, L., Lan, J., Ma, Y.H., Gao, J.M., and Li, Y.L. "Numerical study on heat transfer by swirling impinging jets issuing from a screw-thread nozzle", International Journal of Heat and Mass Transfer, 115, pp. 232-237 (2017).

13. Draksler, M., Koncar, B., Cizelj, L., and Niceno, B. "Large eddy simulation of multiple impinging jets in hexagonal configuration-flow dynamics and heat transfer characteristics", International Journal of Heat and Mass Transfer, 109, pp. 16-27 (2017).

14. Trinh, X.T., Fenot, M., and Dorignac, E. "Flow and heat transfer of hot impinging jets issuing from lobed nozzles", International Journal of Heat and Fluid Flow, 67, pp. 185-201 (2017).
15. Tsaoulidis, D. and Angeli, P. "Liquid-liquid dispersions in intensified impinging-jets cells", Chemical Engineering Science, 171, pp. 149-159 (2017).

16. Charmiyan, M., Azimian, A.R., Shirani, E., Aloui, F., Degouet, C., and Michaelis, D. "3D tomographic PIV, POD and vortex identification of turbulent slot jet flow impinging on a flat plate", International Journal of Mechanical Science and Technology, 31, pp. 5347-5357 (2017).

17. Charmiyan, M., Azimian, A.R., Shirani, E., and Aloui, F. "Capability assessment of five different RANS-based turbulence models to simulate the various regions of slot turbulent impingement jet flow", In $A S M E$ 2017 Fluids Engineering Division Summer Meeting, p. V01BT11A011 (2017).

18. Chacon Rebollo, T. and Lewandowski, R., Mathematical and Numerical Foundations of Turbulence Models and Applications, Springer, pp. 1-517 (2014).

19. Van Leer, B. "Towards the ultimate conservative difference scheme. II. Monotonicity and conservation combined in a second-order scheme", Journal of Computational Physics, 14(4), pp. 361-370 (1974).

\section{Biographies}

Mohammad Ali Modaresi is a PhD Candidate of Mechanical Engineering at Tarbiat Modares University. He received his MSc degree from Isfahan University of Technology in 2016.

Ebrahim Shirani is a Professor of Mechanical Engineering at Isfahan University of Technology, Iran . He received his BS degree from Sharif University of Technology, Iran in 1975 and his MS and PhD degrees from Stanford University, USA in 1977 and 1981, respectively, all in Mechanical Engineering. His research interests include computational fluid dynamics, computational micro and nano-fluid dynamics, turbulence and turbulence modeling, modeling and numerical simulation of interfacial flows, turbomachinery and biofluid dynamics. He is the author of 12 books and has published more than 110 journal papers and several conference papers.

Mahmoud Charmiyan received his $\mathrm{PhD}$ degree from Isfahan University of Technology in 2017. He is currently an Assistant Professor at University of Ayatollah Ozma Boroujerdy, Iran. His research interests include numerical and experimental fluid mechanics and turbulence modeling.

Fethi Aloui has been a Professor of Mechanical Engineering and Energy at the University of Valenciennes and Hainaut-Cambresis (UVHC), France (at the Engineering School ENSIAME), Laboratory LAMIH (UMR CNRS 8201), since September 2011 
after working more than 14 years as an Associate Professor in Process Engineering, Energy and Fluid Mechanics at the University of Nantes, France. His specialty field is transfer and transport phenomena in fluid mechanics and engineering processes. He is the author of 2 books and has published over 200 journal and conference papers so far. He serves as an Associate Editor of the Journal of Applied Fluid Mechanics and the International Journal of Energy Research. He is also the co-organizer (each year since 2010) of the ASME-FEDSM (Fluids Engineering Division Summer Meeting). He regularly reviews papers for International Journal of Energy Research, International Journal of Heat and Fluid Flow, International Journal of Coal Science and Technology, Fuel, Journal of Applied Fluid Mechanics, Applied Energy, and many other journals.

Amine Koched is an Application Engineer at TSI France Inc. He holds a PhD in Fluid Mechanics from University of Nantes in France. He has worked previously as a scientific assistant, PostDoc, at IRSN (French Institute of Radioprotection and Nuclear Safety) and at Ecole des Mines de Douai in France, and he was a lecturer at University of Nantes. Dr. Koched published 9 journal papers and participated in more than 30 international conferences.

Michel Pavageau is the Director of International Affairs at Institut Mines-Télécom, one of France's premier institutes of technology. His mandate is to establish IMT's international strategy and oversee its execution. Before assuming his current post in 2012, he was the Dean of International Affairs at IMT Mines Albi, one of the IMT campuses. He is also a Full Professor of the Ecoles des Mines, specializing in experimental fluid dynamics (Turbulent heat and mass transfers applied to air quality issues including urban air quality and fire propagation in confined areas). He completed his PhD in France (Centrale Nantes) and post-doctoral studies in Germany (Uni. Hamburg). He has written extensively on basic and applied sciences and on pedagogy. Having been the administrative and pedagogical coordinator of the teaching program in Physical Sciences for L3 and M1 level students at IMT Atlantique during 10 years, Michel Pavageau's main focus and struggle has been the idea of overcoming the traditional silos between mathematics and the sciences that utilize them. In this respect, among others, he has developed the learning portal http://epiphys.emn.fr 